\title{
Agronegocios
}

Artículo

\section{El efecto sucesor y el proceso de integración generacional: Temas clave en la sucesión familiar agrícola}

The Successor effect and the Generational Integration Process: key aspects of the family

farm succession

\section{Víctor Rodríguez Lizano ${ }^{1}$ Mercedes Montero Vega²}

Fecha de recepción: 08 de abril, 2020 Fecha de aprobación: 05 de junio, 2020

Vol.6 $\mathrm{N}^{\circ} 2$ Julio- diciembre 2020

Rodríguez, V. y Montero, V.(2020). El efecto sucesor y el proceso de integración generacional: temas clave en la sucesión familiar agricola. Revista e-Agronegocios, 6(2). https://revistas.tec.ac.cr/index.php/eagronegocios/

article/view/5210

DOI: https://doi.org/10.18845/ea.v6i2.5210

${ }^{1}$ Universidad de Costa Rica. Costa Rica. Docente en la Escuela de Economía Agricola y Agronegocios. Correo electrónico: victoantonio.rodriguez@ucr.ac.cr 2Universidad de Costa Rica. Costa Rica.Docente en la Escuela de Economía Agrícola y Agronegocios. Correo electrónico: mercedes.montero@ucr.ac.cr. 


\section{Resumen}

Este estudio examina el efecto sucesor y el Proceso de Integración Generacional (PIG) con el fin de aportar información relevante en el camino de entender mejor la sucesión generacional agrícola. El estudio se llevó a cabo en fincas hortícolas familiares en Zarcero, Costa Rica. Se realizaron 126 encuestas en finca entre enero y mayo de 2019. Para cuantificar el efecto sucesor, se generaron tres perfiles de finca según probabilidad de sucesión (baja, media y alta). Así mismo se cuantificó el comportamiento de la producción para cada finca en los últimos cinco años (disminuido, igual, aumentado). Esto permitió a través una prueba chi cuadrado determinar el comportamiento de la producción de las fincas según su probabilidad de sucesión. Por otro lado, se acotó el PIG a seis acciones claves identificadas en la literatura. Dichas acciones se cuantificaron y se corrieron dos modelos por medio de Mínimos Cuadrados Ordinarios (MCO), en los cuales se utilizaron variables socioeconómicas y psicológicas como factores independientes. Con respecto al efecto sucesor, se encontró que fincas que presentan un sucesor claramente definido (probabilidad de sucesión alta), son más propensas a presentar incrementos de su producción que aquellas que por el contrario no poseen un sucesor claro o cuyo estado de sucesión aún no está definido. Con respecto al PIG, el género del sucesor y variables proxy de riqueza poseen los coeficientes más altos dentro de las variables socioeconómicas que explican el PIG. Se observa que la variable psicológica "intención" es la que mayor correlación presenta con el PIG y una de las que mejor explica el mismo. Se demostró por lo tanto, que poseer un sucesor identificado es clave para la competitividad y sostenibilidad de las fincas familiares. Así mismo, el enfoque tradicionalista donde se trata de explicar el PIG o la sucesión por medio de variables socioeconómicas, arroja valores bajos de explicación $(\mathrm{R} 2: 0,45)$ debido a su enfoque reduccionista. Los hallazgos dan paso a una nueva tendencia de investigación relacionada, donde las variables psicológicas, culturales y la economía conductual parecen ser opciones novedosas y las más atinentes al tema..

Palabras clave: agricultura familiar, horticultura, transferencia intergeneracional, modelaje econométrico, planificación agrícola.

\section{Abstract}

This study examines the successor effect and the Generational Integration Process (GIP) in order to generate relevant information on the way to better understand succession process in agriculture. The study was carried out on horticultural family farms in Zarcero, Costa Rica. 126 interviews were conducted between January and May 2019. In order to quantify the successor effect, three farm profiles were generated according to the probability of succession (low, medium and high). Likewise, it was quantified the behavior of the last five years farm's production (decreased, equal, increased). Through a chi square test it was determine the linkage of the behavior of the production of the farms with the probability of succession. On the other hand, the GIP was quantified using six key actions which were identified amongst the literature. Two Ordinary Least Squares (OLS) models were conducted in which socioeconomic and psychological variables were taken as independent factors. Regarding the successor effect, it was found that farms that have an identified successor (high succession probability), are more likely to show increases in their production during the last five years. The opposite can be observed in farms where succession status is not yet defined. Regarding the GIP, the gender of the successor and proxy variables of wealth, show the highest coefficients within the socioeconomic variables. The psychological variable "intention" shows the highest correlation with the GIP and is one of the explanatory factor that best fit. Having an identified successor is a key factor to improve productivity, competitiveness and sustainability of family farms, likewise, we showed that the traditionalist approach where GIP and succession are tried to be explained by means of socioeconomic variables, yields low explanatory values (R2: 0, 45). This results leaves room to a new strand of related research, where psychological variables and behavioral economics seem to be novel options and the approaches that best suits the subject..

Key words: family farm, horticulture, intergenerational transfer, econometric modelling, agricultural planning. 


\section{Agradecimientos}

Se agradece al Programa de Posgrado en Ciencias Agrícola y Recursos Naturales (PPCARN) y a la Universidad de Costa Rica por otorgar los recursos necesarios para llevar a cabo este trabajo, el cual fue realizado durante los estudios doctorales del autor principal.

\section{Introducción}

Hoy en día existe un envejecimiento generalizado en los agricultores costarricenses, este fenómeno también ocurre en Europa en donde solamente el 11\% de los agricultores está por debajo de 40 años de edad (Eurostat, 2016). A nivel nacional, la edad promedio del agricultor es de 54 años, y el 22,5\% de estos tienen más de 65 años (INEC, 2015), siendo considerados, según la legislación costarricense, como adultos mayores. Dicho envejecimiento ocurre por la poca introducción de jóvenes al sector y la permanencia de los agricultores titulares en sus fincas hasta edades avanzadas, producto de la baja tasa de sucesión que existe en el sector.

La salida de los jóvenes tiene serias implicaciones a lo interno de la finca, a nivel territorial y en la productividad del sector agropecuario. Se ha identificado que la emigración rural de jóvenes puede provocar deterioro cultural en las zonas agrícolas (Matte \& Machado, 2017), además, la no continuación de la actividad por parte de los sucesores genera una pérdida del conocimiento específico relacionado con la finca, provocando mermas en la productividad de la finca en el corto plazo si esta es adquirida por personas ajenas a la familia (Bertoni \& Cavicchioli, 2016b). Así mismo, a agricultores de mayor edad se les asocia una menor adopción de nuevas tecnologías o de esquemas agro-sostenibles (Dabkiene, 2015), por lo que la presencia de agricultores jóvenes es fundamental para la adaptación de las fincas a las condiciones climáticas, tecnológicas y las cambiantes demandas de los mercados actuales. Adicionalmente, se ha demostrado que las fincas que no poseen un sucesor actual o potencial poseen mayores probabilidades de entrar en un periodo de estancamiento o decrecimiento en años previos al retiro del agricultor principal (efecto sucesor) (Inwood \& Sharp, 2012; Wheeler et al., 2012). Se demuestra por tanto, que la permanencia de los jóvenes en las fincas familiares posee implicaciones positivas en la productividad, lo cual es clave para el aseguramiento alimentario del pais y el desarrollo rural.

Tradicionalmente, el enfoque cuantitativo para explicar sucesión agrícola ha utilizado únicamente variables socioeconómica y con estas se han generado modelos de respuesta binaria, no obstante, este enfoque ha presentado bajos niveles de explicación del fenómeno de sucesión y otros relacionados (Fischer \& Burton, 2014). Por otro lado, a nivel cualitativo, se ha identificado que el integrar en actividades de la finca a los hijos desde edades tempranas, puede tener un efecto mayor en el éxito de la sucesión que las condiciones socioeconómicas per se (Fischer \& Burton, 2014). La acción de ir incluyendo a los hijos ha sido definida como el Proceso de Integración Generacional (PIG) (Perrachón, 2016) y hasta ahora solo se ha estudiado de forma cualitativa. Aún y cuando la importancia del PIG ya esté probada, no se han realizado estudios que expliquen cuales variables lo provocan. Si se parte del hecho que el PIG es el 
factor que más influye en la sucesión, entonces, identificar las variables que lo causan puede resultar en un avance clave para orientar acciones de política que mejoren la sucesión familiar agrícola en el país.

El principal objetivo de esta investigación es el de aportar información clave para entender mejor la sucesión generacional agrícola. Dado lo anterior, esta investigación se enfoca en dos puntos fundamentales de la sucesión generacional. El primer tema que se analizó, debido a su implicación en la productividad y eficiencia del sector, es el efecto sucesor, por lo que se comprobó si este se presenta en las fincas hortícolas de Zarcero. Como segundo tema, dada su importancia en la sucesión familiar agricola, se analizan las variables que se correlacionan con el PIG a través de un modelo de mínimos cuadrados ordinarios (MCO), esto con el fin de identificar si variables psicológicas como la "intención" aportan más a la explicación del PIG que variables socioeconómicas tradicionales.

\section{Marco referencial}

El efecto sucesor, también denominado efecto de retiro, hace alusión al comportamiento que presenta una finca en su producción según su condición de sucesión generacional (Potter \& Lobley, 1996). Se ha identificado que fincas sin sucesor definido, entran en un proceso de estancamiento o disminución de su producción y productividad en los años previos al retiro del titular. Sin embargo, el proceso se revierte en fincas que presentan un sucesor claramente definido. En estos casos, en los años previos al retiro del agricultor titular, se observa un aumento en la producción. Este fenómeno ha sido hallado por Calus et al., (2008) y Potter \& Lobley (1996), lo cuales observaron dichos comportamientos en Bélgica e Inglaterra respectivamente.

El otro eje estudiado en esta investigación es el Proceso de Integración Generacional (PIG), según Perrachón (2016), la sucesión familiar agrícola se explica fundamentalmente por el PIG. Este hace referencia al relacionamiento de distintas generaciones de una familia con el fin de traspasar la finca, de esta forma, si dicho proceso de integración es exitoso, van a existir mayores probabilidades de existencia de un sucesor. Si bien Perrachón (2016) proporciona un significado del PIG, este no genera un listado de las acciones que conforman lo conformen. Esta investigación genera un recopilado de acciones que según la literatura se han identificado como fundamentales en el PIG. Estas son:

1- $\quad$ Comunicación entre el agricultor y sus hijos sobre la intención de sucesión: La Universidad Técnica Nacional(UTN) (2015) menciona que uno de los factores que han determinado la salida de los jóvenes es la falta de comunicación clara sobre el proceso de sucesión de la finca; así mismo, Dodero (2006), menciona en su estudio realizado en 12 países de Latinoamérica, que la falta de comunicación es la segunda razón que genera en las empresas familiares problemas de crecimiento o sucesión.

2- Incorporar al sucesor en la toma de decisiones en finca: Gallo and Peluso (2013), men- 
cionan que uno de los factores que más influye en los hijos a emanciparse es la falta de participación en la toma de decisiones en la finca. La falta de incorporación en la toma de decisiones provoca futuros agricultores con pocas capacidades administrativas y pone en peligro la continuidad de la finca (Uchiyama et al., 2008).

3- $\quad$ Otorgarle un pago acorde con las labores que desarrolla en la finca: Mazorra (1999) indica que el reconocerle al sucesor por un medio de pago su trabajo es de suma importancia, en donde el titular utiliza el pago como incentivo para que hijo se mantenga trabajando en la finca, factor que también fue estudiado por May et al. (2019).

4- $\quad$ Otorgarle los recursos necesarios para que el hijo estudie: se parte del hecho que una sucesión exitosa no solamente es llegar a tener un sucesor al mando de la finca en determinado momento, sino que también, dicho sucesor tiene que estar preparado para administrar la finca de manera exitosa. Dirven (2002) indica que la educación formal constituye un capital invaluable y puede generar sinergias con las experiencias adquiridas en técnicas productivas, la gestión del negocio agropecuario, los canales de comercialización y la gestión del crédito.

5- $\quad$ Facilitar un segmento de la finca para que el hijo lo administre de forma independiente: Mesen (2009:87) menciona la importancia de otorgarle a los hijos un segmento de la finca, para que ellos puedan desarrollar sus propios cultivos y administrarlos de la forma más independiente posible. Es una acción que toma el titular con el fin de ir "entrenando" al sucesor, de esta manera, el sucesor va ganando experiencia e inclusive puede llegar a capitalizar suficiente dinero para comprar algún tipo de activo productivo.

6- $\quad$ Planificación: es la generación de un plan de acción para la sucesión. Se considera que una buena planificación en la sucesión empieza mucho antes que la nueva generación esté en edad de poder asumir el control de la finca, la primera acción en la planificación es tener un sucesor identificado (Bjuggren \& Sund, 2001).

Tradicionalmente, los estudios relacionados con sucesión generacional analizan cuatro tipos de variables: variables finca, variables de familia, variables del productor y variables de contexto. Por lo general se relacionan este tipo de variables a través de modelos binomiales tales como logit o probit (Bertoni \& Cavicchioli, 2016a; Suess-Reyes \& Fuetsch, 2016). Seguidamente se realiza una recopilación de estudios relacionados con sucesión generacional según cada tipo de variable.

1.Variables de finca: se ha identificado que fincas con mayores niveles de capital poseen mejores niveles de sucesión (Bertoni \& Cavicchioli, 2016a; Nuthall \& Old, 2017). Así mismo fincas con mayor especialización (Hennessy \& Rehman, 2007; Wolf, 2003) o producción no convencional están correlacionadas con mayores niveles de sucesión (Corsi, 2009; Kerbler, 2012).

2. Variables de familia: ser varón (Cavicchioli et al., 2015; Kerbler, 2008) y venir de una familia de tradición agrícola (Mann, 2007) aumentan las probabilidades de que exista un suce- 
sor en la finca. La educación del sucesor puede jugar un papel a favor si esta está relacionada con la agricultura (Glauben et al., 2009).

3. Variables de productor: la edad por lo general posee un efecto positivo en la probabilidad de sucesión (Mishra \& El-Osta, 2008; Nuthall \& Old, 2017), en donde a mayor edad del titular, mayor es la probabilidad de encontrar un sucesor designado en la finca. Para otras variables, como la escolaridad del titular, existen estudios que muestran tanto efectos positivos (Cavicchioli et al., 2015; Kerbler, 2008; Kimhi \& Nachlieli, 2001) como efectos negativos (Aldanondo-Ochoa et al., 2007; Corsi, 2009; Wolf, 2003).

4. Variables de contexto: se consideran las variables menos estudiadas de las socioeconómicas. Según Corsi (2009) y Kerbler (2012), la afiliación de una finca a una cooperativa influye positivamente sobre la sucesión. Otra variable de contexto como tasa de empleo en la zona ha presentado una s-shape en dos estudios realizados por Bertoni \& Cavicchioli (2016a) y Cavicchioli et al., (2018) respectivamente. Una tercera variable analizada ha sido pagos del gobierno, fincas que poseen transferencias del gobierno presentan una mejor tasa de sucesión (Kerbler, 2008; Mishra \& El-Osta, 2008).

Según Fischer y Burton (2014), el análisis de la sucesión generacional a través de variables socioeconómicas ha demostrado tener un nivel bajo de explicación. Dado lo anterior, podría existir un quinto grupo de variables, las variables psicológicas, las cuales han sido considerablemente menos estudiadas. Para este caso, se utiliza como marco teórico lo expuesto por Ajzen (1991), en donde se menciona que el principal elemento psicológico que define una acción es la intención para hacerla, la cual puede ser cuantificada directamente.

Si bien no existen estudios que traten de entender qué variables provocan el PIG, al ser este parte fundamental de la sucesión generacional agrícola, se tomará el marco teórico anteriormente expuesto, en donde se contemplan cinco tipo de variables socioeconómicas, como base para escoger las variables explicativas del PIG.

Otros estudios analizan la sucesión a través de análisis cualitativos con orientaciones sociológicas. En esta línea, la percepción del trabajo agrícola por parte del productor es una variable determinante en la sucesión familiar, el hecho que el agricultor principal sienta una insatisfacción con el estilo de vida que significa el ser un agricultor, determina la sucesión de la finca según el estudio de Hautaniemi \& Gutmann (2006). Asi mismo, el estudio realizado por Carolan (2018) demuestra que la importancia que se le confiere a "vivir en ambientes rurales", "mantener la finca en manos familiares" y "preservar la finca" pesan más que los aspectos económicos; de esta manera, la sucesión se ve como un proceso que conlleva una fuerte carga emocional del agricultor titular (Conway et al., 2017). Así mismo, el estudio de (Kazakopoulos \& Gidarakou, 2003), hace alusión a aspectos no económicos que determinaron la permanencia de mujeres en la agricultura, dentro de estos aspectos destacan como los más importante "independencia en el trabajo" y "vivir en un ambiente sano". Otros aspectos culturales como el favorecer en la sucesión a solo los hombres (enfoque de género) (Kerbler, 2008) o favorecer al 
hijo mayor con la sucesión de la finca (mayorazgo) (Creighton et al., 2016), son claros ejemplos que la sucesión se ve inmersa en una compleja red de relaciones de diferente índole; por lo que un enfoque cuantitativo puede llegar a establecer un cierto grado de entendimiento, pero está lejos de captar toda la complejidad de la problemática.

Se generan dos tipos de hipótesis: el efecto sucesor está presente en la agricultura familiar hortícola de Zarcero. Como segunda hipótesis se tiene que: el nivel del PIG presente en una finca puede ser explicado tanto por variables socioeconómicas como por variables psicológicas, siendo el aporte de estas últimas significativo.

\section{Metodología}

El estudio se enfocó en fincas pequeñas familiares de carácter hortícola localizadas en Zarcero. Se obtuvo una muestra representativa de los agricultores por medio de la fórmula de proporciones, para la cual se utilizaron los siguientes parámetros: $\mathrm{N}=198$ agricultores de hortalizas mayores a 35 años, $e=5 \%, Z=1,96$ (Significancia de un 95\%), $p=0,76$ (Correspondiente al 76\% de los agricultores de $\mathrm{N}$ que poseen hijos mayores a 15 años) y $\mathrm{q}=0,24$ (1-p). Utilizando estos parámetros se obtiene un tamaño de muestra de 117. Se realizaron visitas a finca, y se lograron obtener 126 encuestas válidas. La encuesta fue de tipo cerrada en donde se cuantificó el nivel del PIG del agricultor titular con cada uno de sus hijos. Se obtuvo una media de 2,74 hijos por productor, lo cual generó una base inicial de datos de 345 (126×2.74) observaciones. Se eliminaron 40 observaciones debido a falta de información, lo cual permitió tener una base final de 305 observaciones completas. La encuesta directa también permitió obtener el estado de sucesión en la finca y la intención del agricultor titular con respecto a que sus hijos se mantengan trabajando en la finca. Cuando se permite, se complementan los resultados con citas de agricultores jóvenes de Zarcero, obtenidas durante el mismo trabajo de campo.

\section{Probabilidad de sucesión en una finca y efecto sucesor}

La probabilidad de sucesión en una finca se recopiló a través de la pregunta abierta directa "¿Qué tan probable es que su hijo/a se haga cargo de la finca en un futuro?" Esta pregunta se analizó de manera cualitativa, lo cual permitió generar una clasificación de tres niveles según la probabilidad de sucesión. Los niveles son:

Nivel 1: probabilidad muy alta. La característica principal corresponde a fincas en las que se tiene al menos un sucesor claramente identificado que trabaja de lleno en la finca. El titular se encuentra totalmente seguro de que al menos un hijo se va a quedar trabajando en la finca, lo cual lo expresan con frases como "estoy 100\% seguro" o "Es totalmente segura la sucesión".

Nivel 2: probabilidad media. La característica principal es que se tiene un sucesor identificado, pero la opinión del agricultor titular es insegura con respecto a la sucesión de la finca, lo cual se denota en expresiones como "Aún no se sabe", "puede ser", "es probable". Por lo 
general, este escenario se observa en fincas que si bien tienen un sucesor identificado, este aún estudia en el colegio o está en la universidad en una carrera no relacionada con el sector agroalimentario o bien tiene un trabajo adicional al trabajo de la finca. También se observa en aquellas fincas que poseen a un sucesor identificado pero mayor a 25 años y que tiene una profesión relacionada con el sector agropecuario, pero además de la finca, posee otro trabajo que le representa la entrada principal.

Nivel 3: probabilidad muy baja. La principal característica de estas fincas es que no poseen un sucesor identificado. Ningún hijo es considerado un sucesor potencial, lo cual se observa con expresiones del agricultor titular como "para nada probable" o "muy poco probable".

Por otro lado, se observó el crecimiento de la producción para cada finca en los últimos cinco años. Esta variable podía tomar tres niveles, ya que la producción podía haberse disminuido, mantenido igual o aumentado. De esta manera, a través de una prueba chi cuadrado, se analizó la dependencia entre el comportamiento de la producción en los últimos cinco años y la probabilidad de sucesión de la finca.

\section{Cuantificación del PIG}

Se recopiló el nivel de PIG que el titular ha llevado con cada uno de sus hijos. El PIG se dividió en seis acciones, de las cuales cuatro se cuantificaron en escala Likert (cinco puntos) y dos de forma binaria. Las acciones cuantificadas en escala Likert son las siguientes: a) acción 1: comunicación entre el agricultor y sus hijos sobre la intensión de sucesión, b) acción 2: nivel de incorporación en la toma de decisiones en finca del sucesor, c) acción 3: grado en que el titular le otorgó los recursos necesarios para que el hijo estudiara y d) acción 4: grado de planificación sobre la sucesión de la finca. Se generó un ítem por acción, en donde uno denota que el agricultor titular no la ha realizado y cinco expresa que se ha realizado la acción de una forma adecuada. Cualquier otro escenario contenido entre estos extremos, tomaría un valor entre dos y cuatro.

Las acciones cuantificadas de forma binaria son las siguientes: a) acción 5: otorgarle un pago acorde con las labores que desarrolla en la finca. (1=Sí, O=No) y b) acción 6: facilitar un segmento de la finca para que el hijo lo administre de forma independiente. ( $\mathrm{Si}=1,0=\mathrm{No})$.

Esta forma de cuantificación admite un PIG mínimo de cuatro puntos y un PIG máximo de 22 puntos por hijo. Debido a que la sumatoria de la acciones del PIG, genera una variable continua discreta, ésta se pudo correlacionar por medio de Mínimos Cuadrados Ordinarios (MCO) con otras variables de índole socioeconómico y con la variable psicológica "intención". A través del algoritmo "Step", en el software R, el cual genera regresiones progresivas probando n cantidad de variables, sus combinaciones y su respectiva correlación con el PIG, se logró encontrar la combinación de variables que maximiza el $\mathrm{R}^{\wedge} 2$ y minimiza otros parámetros tales como el AIC. Se probaron 21 variables socioeconómicas. Luego de este procedimiento, las variables seleccionadas se muestran en el cuadro 1. 
Cuadro 1. Variables explicativas del PIG.

\begin{tabular}{lll}
\hline Variable & Codificación & Detalle \\
\hline Edad & Continua & Edad en años cumplidos del titular. \\
Género & Binaria & Género del hijo. \\
Pensión & Binaria & Si el titular va a recibir pensión del Estado. \\
Ha Producción & Continua & Número de hectáreas en producción. \\
Carro & Binaria & Tenencia de carro exclusivo para la finca. \\
Trabajo & Binaria & Si el hijo trabaja fuera de la finca. \\
Intención & Continua & Deseo expreso del titular por que su hijo/a se \\
\end{tabular}

Para identificar el aporte de la variable psicológica "intención" en la explicación del PIG, se realizaron dos modelos, un primer modelo con la variable "intención" y un segundo modelo con las mismas variables socioeconómicas que el primero pero sin la variable "intención". Se analizó la autocorrelación, heterocedasticidad y multicolinealidad de cada uno de los modelos y se realizaron las correcciones respectivas según los errores robustos de White. Seguidamente los modelos se compararon a través del cambio en el $\mathrm{R}^{\wedge} 2$ ajustado.

\section{Resultados}

\section{Efecto sucesor}

Al analizar los datos verticalmente (cuadro 2), se observa que de aquellas fincas que han disminuido la producción en los últimos cinco años, el 69\% posee una probabilidad de sucesión media o baja y solamente un 31\% poseen una probabilidad alta de sucesión. Sin embargo, dichas proporciones se invierten cuando se trata de fincas que han aumentado su producción en los últimos cinco años; para este caso, la mayor proporción de fincas se encuentra en la probabilidad alta con un $65 \%$, mientras que aquellas que presentan una probabilidad baja de sucesión solo representan un 35\%. Las fincas que han mantenido su producción en los últimos cinco años se encuentran distribuidas de igual forma (50/50) entre aquellas fincas con probabilidad media o baja y alta. 
Cuadro 2. Distribución del comportamiento de la producción total de la finca en los últimos cinco años según probabilidad de sucesión. Lectura vertical

\begin{tabular}{c|cccc}
\cline { 3 - 5 } \multicolumn{2}{c}{} & \multicolumn{3}{c}{$\begin{array}{c}\text { Comportamlento de la producclón total de la flnca en } \\
\text { los últimos clnco años }\end{array}$} \\
\cline { 2 - 5 } \multicolumn{2}{c}{} & Disminuido & lgual & Aumentado \\
\hline $\begin{array}{c}\text { Probabilida } \\
\text { d de } \\
\text { sucesión }\end{array}$ & Media o baja & $24(69 \%)$ & $34(50 \%)$ & $8(35 \%)$ \\
\hline \multicolumn{1}{l}{ Total } & $11(31 \%)$ & $34(50 \%)$ & $15(25 \%)$ \\
\hline
\end{tabular}

Así mismo, se analizaron los datos de forma horizontal (cuadro 3) tomando como referencia la variable probabilidad de sucesión.

Cuadro 3. Distribución del comportamiento de la producción total de la finca en los últimos cinco años según probabilidad de sucesión. Lectura horizontal.

\section{Comportamiento de la producción total de la finca en los últimos cinco años}

\begin{tabular}{l|lcccc}
\multicolumn{2}{c}{} & Disminuido & Igual & Aumentado & Total \\
\hline Probabilidad & Media o baja & $24(36 \%)$ & $34(52 \%)$ & $8(12 \%)$ & 66 \\
de sucesión & Alta & $11(18 \%)$ & $34(57 \%)$ & $15(25 \%)$ & 60 \\
\hline
\end{tabular}

La lectura horizontal del cuadro 3 permite identificar que en el caso de fincas que poseen una probabilidad de sucesión media o baja, el porcentaje que ha aumentado la producción (12\%) es menor que el de aquellas que ha disminuido la producción (36\%). Dicho comportamiento se invierte si se trata de fincas con probabilidad de sucesión alta. Para este caso el porcentaje de fincas que ha aumentado la producción en los últimos cinco años (25\%) es mayor que el de aquella que la han disminuido (18\%). La prueba prueba chi cuadrado arroja un valor calculado de 6,69 con dos grados de libertad. Dicho valor genera un valor p asociado de 0,03, con lo cual se rechaza la hipótesis nula de no dependencia entra las variables analizadas. Se confirma que en aquellas fincas con un sucesor definido es más común observar un proceso de expansión de su producción, lo cual se revierte en casos donde la sucesión no es clara. Lo anterior se refuerza con expresiones de agricultores jóvenes que han sido designados como sucesores y que están actualmente trabajando las fincas junto con sus padres. Algunos testimonios son: 
"Tomo la finca a raíz de buscar un crecimiento financiero, él (su padre) ya lo tiene, entonces él busca estabilidad, entonces ya me toca a mí esforzarme por mi solo (...) Teníamos metas diferentes, la mía era crecer la de él (el papá) era buscar estabilidad." (Agricultor joven 1).

En relación con lo anterior el agricultor 10 apunta:

"(...) ya ahora estamos sembrando más, tenemos más terreno alquilado, entonces se ha ido creciendo, como le digo, uno va agarrando experiencia y puede ir mejorando ciertas cosas y hace que las producciones sean un poco mejores." (Agricultor joven 2).

\section{Modelaje del Proceso de Integración Generacional (PIG)}

Los parámetros obtendios de la ecuación de regresión utilizando el PIG como variable dependiente se muestran en el cuadro 4.

Cuadro 4. Resultados del modelo de regresión multiple I. PIG como variable dependiente

\begin{tabular}{ccccc}
\hline Regresores & Coeficientes & Desv. Std & Valor t & Significancia \\
\hline Intercepto & 12,37824 & 1.18358 & 10,458 & $<2 \mathrm{e}-16^{* \star *}$ \\
Edad & $-0,07606$ & 0,02314 & $-3,287$ & $0,001133^{* *}$ \\
Género & $-2,06044$ & 0,44015 & $-4,681$ & $4,35 \mathrm{e}^{* 06^{* *}}$ \\
Pensión & $-1,5077$ & 0,48733 & $-3,094$ & $0,002165^{* *}$ \\
Ha Producción & 0,1128 & 0,03018 & 3.738 & $0,000223^{* *}$ \\
Carro & 1,42445 & 0,58236 & 2,446 & $0,015028^{*}$ \\
Trabajo & $-0,53834$ & 0,24426 & $-2,204$ & $0,028299^{*}$ \\
Intención & 1,24627 & 0,13053 & 9.548 & $<2 \mathrm{e}-16^{\cdots *}$ \\
\hline
\end{tabular}


Todos los parámetros muestran valores significativos, en donde se identifica el intercepto con un valor de 12,38, se puede denominar este valor como el nivel autónomo de PIG, el cual independientemente de las condiciones socioeconómicas de la finca, el titular va a generar 12,38 puntos.

Se observa que la edad del titular exhibe una relación negativa con el nivel del PIG, lo anterior es un resultado intuitivo en el tanto que a mayor edad posea un agricultor, menor nivel de PIG va a realizar el agricultor con sus hijos.

Género es la variable que mayor ingerencia posee en el nivel del PIG. Las mujeres poseen en promedio 2,06 puntos menos de PIG que los hombres. Se observa en campo que la finca por lo general no es una opción para la mujeres, por lo que estas se dedican a otras actividades o inclusive dejan Zarcero en busqueda de otras opciones de trabajo. Lo anterior se sustenta con expresiones tales como:

"En un momento tomamos la decisión de apoyar a nuestras hermanas que eran las que estaban con carreras universitarias entonces diay nosotros esforzarnos aqui trabajando en la finca para que ellas estudiaran en San José." (Agricultor 12).

También se observa que las mujeres por lo general no se involucran en la siembra como tal, pero en el mejor de los casos se relacionan indirectamente en actividades relacionadas, tales como aportar valor agregado a los productos generados en la finca. La siguiente expresión sustenta lo expuesto:

"Bueno primero empecé yo con él y trabajamos como socios. Una hermana de nosotros, ella se fue a estudiar y la otra se incorporó con nosotros. Entonces ella se ha metido con la parte de procesados y cosas de los mismos productos que salen de la finca. Ella hace mermeladas con las frutas, entonces ya ella se pasó para otro lugar pero siempre apoyándonos." (Agricultor 20).

Los resultados indican que, el hecho que el titular vaya a tener una pensión por parte del Estado, disminuye el nivel de PIG medio en una finca en 1,5 puntos.

Variables como la tenencia de vehículo exclusivo para el uso de la finca y cantidad de hectareas de producción, son variables proxy para medir la riqueza del agricultor. En ambos casos, el signo obtenido está en concordancia con lo esperado, en donde productores con mayor nivel de riqueza presentaron mayor nivel de PIG.

Por último, se tomó en cuenta la variable intención del agricultor para que el hijo/a se mantuviera trabajando en la finca, para este caso, esta se considera una variable psicológica y posee el segundo impacto positivo más alto de todas la variables analizadas. Por cada punto que aumenta la intención (variable medida en 5 puntos), el sucesor aumenta el PIG en 1,25 puntos en promedio. Seguidamente se corrió un modelo II sin la variable intención, con el principal 
propósito de evidenciar el aporte de esta variable psicológica a la explicación del PIG. Los resultados se muestran en el cuadro 5.

Cuadro 5. Resultados del modelo de regresión multiple II. PIG como variable dependiente.

\begin{tabular}{ccccc}
\hline Regresores & Coeficientes & Desv. Std & Valor t & Significancia \\
\hline Intercepto & 15,78357 & 1,28852 & 12,249 & $<2 \mathrm{e}^{*} 16^{* \star *}$ \\
Edad & $-0,06223$ & 0,02636 & $-2,361$ & $0,0189^{*}$ \\
Género & $-3,60846$ & 0,4672 & $-7,724$ & $1,75 \mathrm{e}^{*}-13^{* *}$ \\
Pensión & $-0,98798$ & 0,55292 & $-1,787$ & $0,0750$. \\
Ha_Producción & 0,15085 & 0,03416 & 4,417 & $1,41 \mathrm{e}^{* 0} 5^{\cdots}$ \\
Carro & 1,2253 & 0,66447 & 1,844 & $0,0662$. \\
Trabajo & $-0,59515$ & 0,2788 & $-2,135$ & $0,0336^{*}$ \\
\hline
\end{tabular}

Nota. Significancia: $0^{\prime * \star * \prime} 0.001^{1 * * \prime} 0.01^{\prime * \prime} 0.05$ ". $0.1^{\prime \prime 1}$

Para el caso del modelo II todas las variables exhiben los mismos signos presentados en el modelo I. La idea de generar un segundo modelo es comparar el ajuste de los mismos tomando en cuenta la ausencia de la variable psicológica "intención" en el modelo II. Dicha comparación se realizó a través del contraste del valor $\mathrm{R}^{\wedge} 2$ ajustado para ambos modelos. En el cuadro 6 se muestran los indicadores de ajuste de ambos modelos.

Cuadro 6. Indicadores relacionados a ambos modelo de regresión multiple. PIG como varaible depen

\begin{tabular}{ccccc}
\hline Indicadores & $\begin{array}{c}\text { Valor } \\
\text { Modelo I }\end{array}$ & Significancia & $\begin{array}{c}\text { Valor } \\
\text { Modelo II }\end{array}$ & Significancia \\
\hline $\mathrm{R}^{2}$ ajustado & 0,45 & & 0,28 & \\
Estadistico F & 36,71 & $<2,2 \mathrm{e}-16$ & 21,2 & $<2,2 \mathrm{e}-16$ \\
Durbin-Watson & 1,94 & 0,299 & 1,94 & 0,282 \\
Breusch-Pagan & 10,573 & 0,158 & 9,001 & 0,1735 \\
Jarque-Bera & 1,303 & 0,521 & 5,213 & 0,074 \\
\hline
\end{tabular}


Para ambos modelos, los estadisticos $\mathrm{F}$ poseen valores $\mathrm{p}$ menores a 0,05 validando la significancia general en los dos casos. Del modelo I al II se observa una disminución de 0,45 a 0,28 en el R2 ajustado. Así mismo, luego de las correcciones aplicadas, ambos modelos muestran, estadisticos Durbin-Watson cercanos a 2 denotando la no presencia de autocorrelación. Por medio de la corrección de errores robustos de White, para ambos modelos se logra no recharzar la presencia de homocedasticidad de los residuos.

\section{Discusión}

Tanto la lectura vertical como horizontal de los datos relacionados con el efecto sucesor, admiten que existe una relación de dependencia entre el comportamiento de la producción de la finca en los últimos cinco años y la probabilidad de sucesión. Fincas que presentan un sucesor claramente definido (probabilidad de sucesión alta), son más propensas a presentar incrementos de su producción que aquellas que por el contrario no poseen un sucesor claro o cuyo estado de sucesión aún no está definido. Lo anterior puede deberse a que agricultores próximos a retirarse no van a estar en disposición de invertir en nuevas tecnologías, tierra o bienes de capital; ya que el pago de este tipo de activos por lo general se da a largo plazo, por lo que el agricultor mayor no va a poder gozar de los beneficios de dichas inversiones. Así mismo, un agricultor que no posee un sucesor definido puede tender a desintensificar su producción debido a la venta de activos de capital o tierra, lo cual se verá reflejado en su producción.

Con respecto al PIG, se observa una disminución del $\mathrm{R}^{\wedge} 2$ ajustado de un 0,45 a un 0,28 entre el modelo I y el modelo Il; esto debido a la omisión de la variable "intención". Esta disminución representa un $37 \%$ menos de explicación de la varianza del PIG. Contrario a lo que se podría pensar, la variable "intención" no posee una correlación alta con las variables socioeconómicas expuestas en los modelos, esta presenta una correlación promedio de 0,084. Sin embargo, la "intención" posee una correlación de 0,55 con la variable PIG, lo cual indica que las variables que afectan la "intención" del agricultor por incluir a su hijo en el trabajo agrícola no dependen del nivel socioeconómico, sino más bien de otros tipos de variables psicológicas. La teoría psicológica expuesta por Ajzen (1991), expone que la intención es la principal variable que afecta la ejecución de una acción (en este caso el PIG es la acción). El mismo autor, en su Teoría del Comportamiento Planeada (TCP), expone que existen tres componentes que explican la intención de una persona hacia una acción, las cuales son: (1) Actitud hacia el Comportamiento (AC): el grado con que el individuo está a favor o en contra de un determinado comportamiento. En esta influyen las creencias que posea el individuo. (2) Normas sociales o aspectos normativo (AN): presión social para llevar a cabo un comportamiento determinado, relacionado con las expectativas de otras personas y (3) Control del Comportamiento Percibido (CCP): qué tan fácil o difícil piensa una persona que le va a ser llevar a cabo una actividad.

El PIG se puede ver también como una cuestión de género, en donde se favorece a los hombres sobre la mujeres, lo anterior se demuestra en que los coeficientes de género para ambos modelos son los más altos en términos absolutos, lo cual denota que los hombre poseen en promedio un nivel mayor de integración en la finca que las mujeres. Esto abre una puerta para 
la discusión y reflexion sobre equidad de genéro en el sector agropecuario y en la integración de mujeres a las labores agricolas, lo cual, según los resultados obtenidos es desigual por el momento.

Así mismo, se debe explorar la introducción de variables de índole de negocios, como por ejemplo la cultura empresarial que presenta el titular. Agricultores que no ven su finca como un negocio pueden afectar de manera negativa en la percepción de su hijo sobre la visualización de la finca como emprendimiento viable del cual depender económicamente, lo cual es un problema que se puede seguir arrastrando de generación en generación tal y como lo expone (Couzy \& Dockes, 2008). Este tipo de variables puede que tengan correlación con el nivel del PIG que genera un agricultor titular con sus sucesores.

La falta de ver en la finca un negocio viable puede ser producto del efecto de diversas variables, las cuales pueden incidir directamente en que el joven sucesor abandone la finca. Aspectos como la educación formal que posea el titular de la finca (relacionada positivamente con la capacidad de gestión) y el enfoque del sistema de educación actual donde se da orientación a ser empleado y no a ser empleador (Camarena Gómez \& Velarde Hernández, 2009), son dos ejemplos de una amplia gama de variables que podrían afectar el complejo proceso de sucesión.

Los resultados exponen que los agricultores titulares que poseerán pensión, generan un menor nivel de integración en sus hijos. Al respecto Grubbström \& Sooväli-Sepping, (2012) mencionan que el poseer una pensión que no satisfaga las necesidades del agricultor de forma completa, posee un efecto negativo en la integración y por lo tanto en la sucesión de la finca, esto debido a que el agricultor necesita seguir cultivando la finca para complementar los ingresos. Dichos resultados están en línea con los encontrados en esta investigación.

En contraposición a los resultados encontrados, Uchiyama et al., (2008) exponen que el no tener pensión puede significativamente retardar la sucesión. Los agricultores mayores no quieren confiar su futuro en agricultores más jóvenes y por lo tanto cuesta que estos deleguen la administración de la finca. Para el caso de esta investigación, la pensión fue una variable dicotómica, por lo que no se pudo ahondar en la magnitud de la misma, sin embargo, se hipotetiza que estamos en la presencia del escenario expuesto por Grubbström \& Sooväli-Sepping, (2012). Lo anterior explicaría el signo negativo en la variable pensión.

Los resultados de las variables proxy de riqueza (Ha_Producción y carro), se encuentran en línea con los resultados de (Bertoni \& Cavicchioli, 2016a; Glauben et al., 2004; Hennessy \& Rehman, 2007; Kerbler, 2012; Zou et al., 2018), en donde a mayor nivel de riqueza mejor nivel de involucramiento de la siguiente generación.

Dentro de los resultados, se observa una correlación positiva entre la cantidad de hectáreas que posea una finca y el nivel de PIG que realiza un agricultor titular con su sucesor, por lo que se denota la importancia del recurso tierra en el proceso de sucesión generacional. Sin 
embargo, al día de hoy Costa Rica no cuenta con créditos diferenciados para los jóvenes con el fin de acceder a tierras. Plataformas como las que presenta el Inder, a través de la caja agraria, pueden ser una posibilidad de oferta de crédito de esta naturaleza, ya que entre otras poblaciones objetivo, este se orienta a microproductores (Instituto de Desarrollo Rural, 2017), por lo que no limita a jóvenes agricultores para acceder a este tipo de créditos. Así mismo, el Sistema de Banca para el Desarrollo (SBD), el cual para el 2018 colocó el 45\% de sus fondos en crédito agropecuario (SBD, 2018), puede resultar en una posibilidad real de crédito diferenciado para el agricultor joven.

\section{Conclusiones}

La introducción de los jóvenes en la agricultura costarricense es un punto clave para elevar la productividad del sector, mantener la producción agrícola ante el aumento de la población mundial y conservar el capital cultural de las zonas rurales. Tal como se evidenció en esta investigación, el hecho de tener un sucesor claramente identificado, es un factor determinante para el agricultor titular en cuanto a la decisión de intensificar o desintensificar la producción de la finca en los años próximos a su retiro.

Es necesario cambiar el abordaje con que se estudian los temas de sucesión generacional y del Proceso de Integración Generacional (PIG). Se evidencia que las variables socioeconómicas contribuyen poco al entendimiento del PIG, sin embargo este es el enfoque sobre el cual se encuentra la mayor cantidad de estudios. Por otro lado, se observa que la variable psicológica "intención", es la que mayor aporta al entendimiento del PIG. Lo anterior demuestra que el PIG, si bien es influido (poco) por las condiciones socioeconómicas, los factores que mayor pesan son aquellos psicológicos, sobre los cuales existen pocas investigaciones. La "intención" posee una alta correlación con el PIG. Dado lo anterior, futuros estudios relacionados con el tema, deberían de enfocarse en los elementos que la explican, tomando como base la Teoría del Comportamiento Planeado. Esto permitiría entender que tanto peso poseen: a) los aspectos sociales, b) la actitud y c) el control del comportamiento percibido, en la decisión del agricultor titular de llevar un PIG elevado con sus hijos. Este tipo de abordaje empezaría a tener elementos de economía conductual. Dado lo anterior, un enfoque mixto tradicional-conductual puede ser el que mejor se ajuste a este fenómeno. Partiendo del hecho que el PIG es clave en la sucesión de una finca, los resultados de dichos estudios podrían ayudar a dar un salto cuantitativo en el entendimiento de la sucesión generacional y así evolucionar del enfoque tradicional reduccionista en donde se trata de explicar procesos complejos como el PIG a través de variables meramente socioeconómicas. 


\section{Literatura citada}

Ajzen, I. (1991). The theory of planned behavior. Organizational Behavior and Human Decision Processes, 50(2), 179-211. https://doi.org/10.1016/0749-5978(91)90020-T

Aldanondo-Ochoa, A. M., Casanovas, V., \& Almansa, C. (2007). Explaining farm succession: The impact of farm location and off-farm employment opportunities. Spanish Journal of Agricultural Research, 5(2), 214-225. https://doi.org/10.5424/sjar/2007052-241

Bertoni, D., \& Cavicchioli, D. (2016a). Process description, qualitative analysis and causal relationships in farm succession. CAB Reviews Perspectives in Agriculture Veterinary Science Nutrition and Natural Resources, 2016, 1-11. https://doi.org/10.1079/PAVSNNR201611043

Bertoni, D., \& Cavicchioli, D. (2016b). Farm succession, occupational choice and farm adaptation at the rural-urban interface: The case of Italian horticultural farms. Land Use Policy, 57, 739-748. https://doi.org/10.1016/j.landusepol.2016.07.002

Bjuggren, P.-O., \& Sund, L.-G. (2001). Strategic Decision Making in Intergenerational Successions of Small- and Medium-Size Family-Owned Businesses. Family Business Review, 14(1), 11-23. https://doi.org/10.1111/j.1741-6248.2001.00011.x

Calus, M., Van Huylenbroeck, G., \& Van Lierde, D. (2008). The Relationship between Farm Succession and Farm Assets on Belgian Farms. Sociologia Ruralis, 48(1), 38-56. https://doi.or$\mathrm{g} / 10.1111 / \mathrm{j} .1467-9523.2008 .00448 . x$

Camarena Gómez, B. O., \& Velarde Hernández, D. (2009). Educación superior y mercado laboral: Vinculación y pertinencia social ¿Por qué? y ¿Para qué? Estudios sociales (Hermosillo, Son.), 17(SPE), 105-125.

Carolan, M. (2018). Lands changing hands: Experiences of succession and farm (knowledge) acquisition among first-generation, multigenerational, and aspiring farmers. Land Use Policy, 79, 179-189. https://doi.org/10.1016/j.landusepol.2018.08.011

Cavicchioli, D., Bertoni, D., \& Pretolani, R. (2018). Farm succession at a crossroads: The interaction among farm characteristics, labour market conditions, and gender and birth order effects. Journal of Rural Studies, 61, 73-83. https://doi.org/10.1016/j.jrurstud.2018.06.002

Cavicchioli, D., Bertoni, D., Tesser, F., \& Frisio, D. G. (2015). What Factors Encourage Intrafamily Farm Succession in Mountain Areas? Mountain Research and Development, 35(2), 152-160. https://doi.org/10.1659/MRD-JOURNAL-D-14-00107.1

Conway, S. F., McDonagh, J., Farrell, M., \& Kinsella, A. (2017). Uncovering obstacles: The exercise of symbolic power in the complex arena of intergenerational family farm transfer. 
Journal of Rural Studies, 54, 60-75. https://doi.org/10.1016/j.jrurstud.2017.06.007

Corsi, A. (2009). Family farm succession and specific knowledge in Italy. Rivista Di Economia Agraria, 64(1/2), 13-30. CABDirect.

Couzy, C., \& Dockes, A. C. (2008). Are farmers businesspeople? Highlighting transformations in the profession of farmers in France. International Journal of Entrepreneurship and Small Business, 6(3), 407.

Creighton, J., Blatner, K. A., \& Carroll, M. S. (2016). For the Love of the Land: Generational Land Transfer and the Future of Family Forests in Western Washington State, USA. Small-Scale Forestry, 15(1), 1-15. https://doi.org/10.1007/s11842-015-9301-2

Dabkiene, V. (2015). Factors affecting the farm sustainability in Lithuania. Ekonomika APK, 4 , 93-100. http://eapk.org.ua/sites/default/files/eapk/15_04_93-100.pdf

Dirven, M. (2002). Las prácticas de herencia de tierras agrícolas: ¿una razón de más para el éxodo de la juventud? CEPAL. https://repositorio.cepal.org//handle/11362/4525

Dodero, S. (2006). Investigación sobre la Empresa Familiar en Latinoamérica (p. 17). Instituto de la Empresa Familiar, ADEN Business School. http://biblioferrersalat.com/media/documentos/Investigacion\%20sobre\%20la\%20Empresa\%20Familiar\%20en\%20Latinoamerica\%20 (2).pdf

Eurostat. (2016). Farm structure survey 2016. https://europa.eu/rapid/press-release_STAT18-4298_en.htm

Fischer, H., \& Burton, R. J. F. (2014). Understanding Farm Succession as Socially Constructed Endogenous Cycles. Sociologia Ruralis, 54(4), 417-438. https://doi.org/10.1111/soru.12055

Gallo, A., \& Peluso, I. (2013). ESTRATEGIAS SUCESORIAS EN LA GANADERÍA FAMILIAR: UN ENFOQUE DE GÉNERO. 13.

Glauben, T., Petrick, M., Tietje, H., \& Weiss, C. (2009). Probability and timing of succession or closure in family firms: A switching regression analysis of farm households in Germany. Applied Economics, 41(1), 45-54. https://doi.org/10.1080/00036840601131722

Glauben, T., Tietje, H., \& Vogel, S. (2004). Farm succession patterns in Northern Germany and Austria-A survey comparison. Department für Wirtschafts- und Sozialwissenschaften der Universität für Bodenkultur.

Grubbström, A., \& Sooväli-Sepping, H. (2012). Estonian family farms in transition: A study of intangible assets and gender issues in generational succession. Journal of Historical Geogra- 
phy, 38(3), 329-339. https://doi.org/10.1016/j.jhg.2012.03.001

Hautaniemi, S., \& Gutmann, M. P. (2006). Land Use and Transfer Plans in the U.S. Great Plains. Great Plains Research: A Journal of Natural and Social Sciences. http://digitalcommons. unl.edu/greatplainsresearch/844

Hennessy, T. C., \& Rehman, T. (2007). An Investigation into Factors Affecting the Occupational Choices of Nominated Farm Heirs in Ireland. Journal of Agricultural Economics, 58(1), 61-75. https://doi.org/10.1111/j.1477-9552.2007.00078.x

INEC. (2015). VI Censo Nacional Agropecuario. Tomo 4: Resultados generales. INEC.

Instituto de Desarrollo Rural. (2017). Plan Nacional de Desarrollo Rural Territorial 20172022. Inder. https://www.inder.go.cr/acerca_del_inder/politicas_publicas/documentos/PNDRT-2017-2022.pdf

Inwood, S., \& Sharp, J. (2012). Farm persistence and adaptation at the rural-urban interface: Succession and farm adjustment. Journal of Rural Studies, 28, 107-117. https://doi.org/10.1016/j.jrurstud.2011.07.005

Kazakopoulos, L., \& Gidarakou, I. (2003). Young women farm heads in Greek agriculture: Entering farming through policy incentives. Journal of Rural Studies, 19(4), 397-410. https://doi. org/10.1016/S0743-0167(03)00022-6

Kerbler, B. (2008). The influence of factors of the socio-geographical structure of mountain farms in Slovenia upon farm succession statuses and decisions. Acta Geographica Slovenica, 48(2), 277-303. https://doi.org/10.3986/AGS48203

Kerbler, B. (2012). Factors affecting farm succession: The case of Slovenia. Agricultural Economics / Zemedelska Ekonomika, 58(6), 285-298.

Kimhi, A., \& Nachlieli, N. (2001). Intergenerational Succession on Israeli Family Farms. Journal of Agricultural Economics, 52(2), 42-58. https://doi.org/10.1111/j.1477-9552.2001.tbo0924.x

Mann, S. (2007). Understanding Farm Succession by the Objective Hermeneutics Method. Sociologia Ruralis, 47(4), 369-383. https://doi.org/10.1111/j.1467-9523.2007.00442.x

Matte, A., \& Machado, J. A. D. (2017). Tomada de decisão e a sucessão na agricultura familiar no sul do Brasil. Revista de Estudos Sociais, 18(37), 130-151. https://doi.org/10.19093/res. v18i37.3981

May, D., Arancibia, S., Behrendt, K., \& Adams, J. (2019). Preventing young farmers from leaving the farm: Investigating the effectiveness of the young farmer payment using a behaviou- 
ral approach. Land Use Policy, 82, 317-327. https://doi.org/10.1016/j.landusepol.2018.12.019

Mazorra, Á. P. (1999). La remuneración del trabajo en la agricultura familiar española (19851997). Investigaciones Geográficas, O(22), 129-147. https://doi.org/10.14198/INGEO1999.22.06

Mesen, R. (2009). Tesis Doctoral La situacion educativa y ocupacional de los jovenes rurales y su implicacion para la economia familiar la sostenibilidad. http://www.academia. edu/4109404/Tesis_Doctoral_La_situacion_educativa_y_ocupacional_de_los_jovenes_rurales_y_su_implicacion_para_la_economia_familiar_la_sostenibilidad

Mishra, A. K., \& El-Osta, H. S. (2008). Effect of agricultural policy on succession decisions of farm households. Review of Economics of the Household, 6(3), 285-307. https://doi. org/10.1007/s11150-008-9032-7

Nuthall, P. L., \& Old, K. M. (2017). Farm owners' reluctance to embrace family succession and the implications for extension: The case of family farms in New Zealand. The Journal of Agricultural Education and Extension, 23(1), 39-60. https://doi.org/10.1080/1389224X.2016.1200992

Perrachón, J. (2016). Una Integración Generacional exitosa permite un Relevo planificado. Plan Agropecuario, 158, 24.

Potter, C., \& Lobley, M. (1996). The Farm Family Life Cycle, Succession Paths and Environmental Change in Britain's Countryside. Journal of Agricultural Economics, 47(1-4), 172-190. https://doi.org/10.1111/j.1477-9552.1996.tboo683.x

SBD. (2018). Informe Anual SBD 2018. https://www.sbdcr.com/sbd-resources-ViewController-context-root/sbd/documentos/Informe\%20Anual\%20SBD\%202018.pdf

Suess-Reyes, J., \& Fuetsch, E. (2016). The future of family farming: A literature review on innovative, sustainable and succession-oriented strategies. Journal of Rural Studies, 47(Part A), 117-140. https://doi.org/10.1016/j.jrurstud.2016.07.008

Uchiyama, T., Lobley, M., Errington, A., \& Yanagimura, S. (2008). Dimensions of Intergenerational Farm Business Transfers in Canada, England, the USA and Japan. The Japanese Journal of Rural Economics, 10, 33-48. https://doi.org/10.18480/jjre.10.33

UTN. (2015). Decano de la Sede Atenas impulsa programa de Integración y Relevo Generacional en el sector agropecuario nacional | Universidad Técnica Nacional I Costa Rica. Universidad Ténica Nacional. http://www.utn.ac.cr/content/decano-de-la-sede-atenas-impulsa-programa-de-integraci\%C3\%B3n-y-relevo-generacional-en-el-sector

Wheeler, S., Bjornlund, H., Zuo, A., \& Edwards, J. (2012). Handing down the farm? The increasing uncertainty of irrigated farm succession in Australia. Journal of Rural Studies, 28(3), 
266-275. https://doi.org/10.1016/j.jrurstud.2012.04.001

Wolf, C. A. (2003). The Influence of Financial, Production and Operator Characteristics on Dairy Farm Transfer Plans. Journal of the ASFMRA, 2003, 1-5.

Zou, B., Mishra, A. K., \& Luo, B. (2018). Aging population, farm succession, and farmland usage: Evidence from rural China. Land Use Policy, 77, 437-445. https://doi.org/10.1016/j.landusepol.2018.06.001 